\section{Atrkivoc

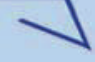

Archive for

Organic Chemistry
The Free Internet Journal

for Organic Chemistry
Paper

Arkivoc 2017, part ii, 149-161

\title{
Synthesis of 5-hetaryluracil derivatives via 1,3-dipolar cycloaddition reaction
}

Dominika Jakubiec, ${ }^{a}$ Łukasz Przypis, ${ }^{b}$ Jerzy W. Suwiński, ${ }^{c}$ and Krzysztof Z. Walczak*b

${ }^{a}$ Selvita S.A. Park Life Science, Bobrzyńskiego 14, 30-348 Kraków, Poland

${ }^{b}$ Department of Organic Chemistry, Bioorganic Chemistry and Biotechnology,

Silesian University of Technology, Krzywoustego 4, 44-100 Gliwice, Poland

${ }^{c}$ Centre of Polymer and Carbon Materials, Polish Academy of Sciences,

M. Curie-Skłodowskiej 34, 41-819 Zabrze, Poland

E-mail: krzysztof.walczak@polsl.pl

This paper is dedicated to Jacek Młochowski - a good colleague and a friend on the occasion of his $80^{\text {th }}$ birthday

Received 06-24-2016

Accepted 08-16-2016

Published on line $08-29-2016$

\section{Abstract}

1,3-Dipolar cycloaddition is a convenient method for construction of various heterocyclic systems. We applied this method for the synthesis 5-hetaryluracil derivatives where substituted uracils played the role of 1,3dipoles or dipolarophiles. Treatment of the nitrile oxide derived from 5-formyluracil and substituted alkenes gave the appropriate 5-(4,5-dihydroisoxazol-3-yl)pyrimidine-2,4(1H,3H)-diones, which by oxidation with $N$ bromosuccinimide were transformed into appropriate 5-(isoxazol-3-yl)uracils. When 5-cyanouracil was used as a dipolarophile in the reaction with nitrile oxides, generated from aromatic aldoximes, several 5-(1,2,4-oxadiazol-5-yl)uracils were obtained. An alternative reaction of 5-formyluracil with an excess of nitriles in the presence of cerium ammonium nitrate as an oxidant gave 1,2,4-oxadiazol-3-yl derivatives in moderate yields.
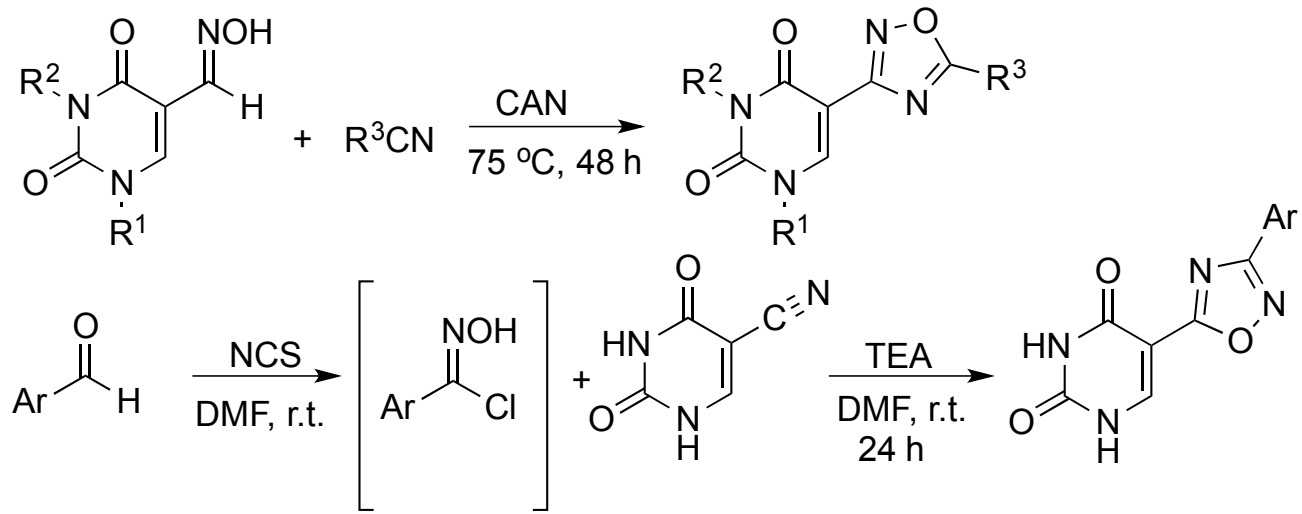

Keywords: Uracil, 1,3-dipolar cycloaddition, 5-cyanouracil, nitrile, nitrile oxides, oxidation 


\section{Introduction}

5-Substituted uracil derivatives and their nucleosides play an important role in medical treatment. Several uracil derivatives exhibit antitumor, antiparasitic and antifungal activity. ${ }^{1-3}$ Promising bioactivity has been observed also in the case of 5-hetaryluracil derivatives. They exhibit anticancer ${ }^{4-9}$ and antiviral activity against several DNA and RNA viruses. ${ }^{10-37}$ The activity against Leishmania donovani promastigotes has also been reported. ${ }^{38,39}$ The heterocyclic ring can be bound to a uracil ring using different strategies. One of the first successful syntheses of 5-hetaryluracils was the preparation of 5-(2,5-dimethylpyrrol-1-yl)uracil by condensation of 5-aminouracil hydrochloride with 2,5-hexanedione. ${ }^{40}$ The same strategy was applied to obtain 5-pyrrol-1-yl-2'-deoxyuridine ${ }^{41}$ or substituted imidazolyl derivatives of uracil. 5-(Imidazol-2-yl)uracil has been obtained in poor yield under Debus-Radziszewski reaction conditions from 5-formyluracil, glyoxal and ammonia. ${ }^{42}$ Formamide-treated with 5-bromoacetyluracil at elevated temperature produced 5-(imidazol-4yl)uracil. ${ }^{41}$ Similarly 5 -bromoacetyluracil reacted with thioamides or thioureas gave 5 -(thiazol-4-yl)uracils in yields over $80 \%{ }^{41,42}$

In another approach, cross-coupling reactions in the presence of an organometallic catalyst have been applied in the synthesis of 5-hetaryluracil derivatives. Thus, uracil or uridine containing a halogen atom on C5, usually iodine or bromine, were treated with appropriate organostannanes derived from different heterocycles like furan, thiophene, pyridine in the presence of transition metal complexes producing 5-hetaryl derivatives in satisfactory yields. ${ }^{43-45}$ Other types of cross-coupling reaction involving an organometallic catalyst applied in the syntheses of 5 -hetaryl derivatives have recently been reviewed. ${ }^{46}$ The less explored strategy for the synthesis of 5-hetaryl uracil derivatives involves 1,3-dipolar cycloaddition reactions. Possible manipulation of dipolarophiles (unsaturated compounds like alkenes, alkynes or nitriles) and 1,3-dipoles (i.e. azides, nitrones etc.,) has made this reaction a very powerful tool for the synthesis of 5 -membered heterocyclic rings. ${ }^{46-54}$

Several papers have described the formation of 5-(1,2,3-triazol-4-yl)uracil derivatives from 5ethynyluracil and organic azides in the presence of Cul-sodium ascorbate catalytic system. ${ }^{46-51}$ The formation of both possible regioisomers 1,4- and 1,5-disubstituted triazoles, depending on the applied catalyst, has been found. Thus, the use of the copper salt-sodium ascorbate system as a catalyst has led to the formation of 1,4regioisomers, whereas 1,5-regioisomers were obtained with $\mathrm{Cp} * \mathrm{RuCl}\left(\mathrm{PPh}_{3}\right)_{2}$ employed instead of the standard $\mathrm{Cu}$ catalyst. ${ }^{35}$ Other 1,3 -dipoles have been used less frequently. The addition of nitrile oxides to 2 '-deoxy-5ethynyluridine was used in the construction of 5-(isoxazol-5-yl) derivatives. ${ }^{52}$ The nitrile oxide and nitrones derived from 5 -formyluracil were applied as 1,3-dipoles in construction of 5-hetaryluracils. ${ }^{53,54}$ Other methods like Diels-Alder cycloaddition, cleavage of diazonium salts formed from 5 -aminouracil in the presence of azoles have had limited application for single reactions only. ${ }^{46}$

In this paper we report the results of our investigation on the 1,3-dipolar addition reaction where uracil derivatives were used either as dipolarophiles or as 1,3-dipoles. Depending on the reagents used, uracil derivatives bearing a heteroaromatic ring on carbon $\mathrm{C} 5$ were obtained directly or the primary forming cycloadduct was oxidized using available oxidants to achieve the desired product.

\section{Results and Discussion}

The key substrates 5 -formyluracils $\mathbf{3}$ and $\mathbf{6}$ were obtained in a sequence of reactions (Scheme 1). To avoid interaction of protons present in the uracil molecule 1 with reactants used in the next steps an uracil derivative was primary alkylated either on both ring $\mathrm{N}$ atoms or only on $\mathrm{N} 1$. Uracil $\mathbf{1}$ was alkylated with 
dimethyl sulfate under basic conditions and the resulting 1,3-dimethyl uracil 2 was formylated under Vilsmeier-Haack reaction conditions. ${ }^{55}$

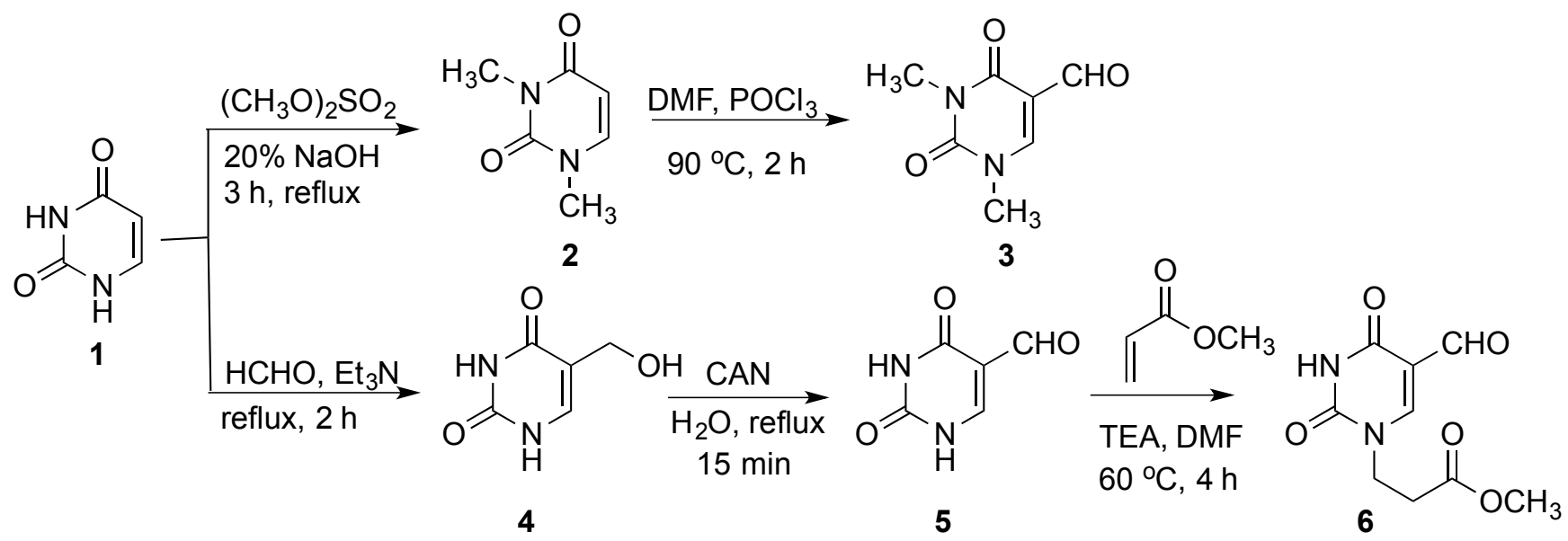

Scheme 1. Synthesis of 5-formyl uracil derivatives.

In a parallel experiment, uracil 1 at first was treated with formaldehyde in the presence of triethylamine and the resulting 5-hydroxymethyluracil 4 was oxidized by ceric ammonium nitrate (CAN) ${ }^{56}$ to 5 formyluracil 5. In both cases the product was obtained in a satisfactory yield exceeding $70 \%$ (Scheme 1). Compound $\mathbf{5}$ was alkylated by methyl acrylate in the presence of triethylamine as a base and product $\mathbf{6}$ was achieved in $80 \%$ yield. ${ }^{56}$ Uracil derivatives 3 and 6 were transformed into appropriate oximes 7a, b in the reaction with hydroxylamine hydrochloride and sodium acetate in aqueous ethanol solution (Scheme 2).

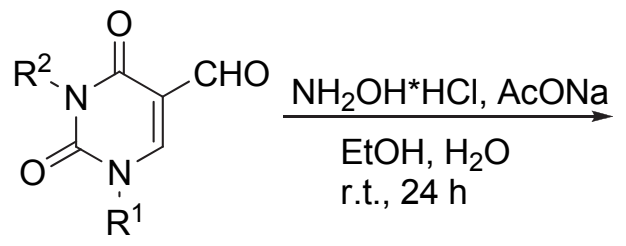

3,6<smiles>[R]n1cc(C=N)c(=O)n([R])c1=O</smiles>

3, 7 a R ${ }^{1}, \mathrm{R}^{2}=\mathrm{CH}_{3}$

6, 7 b R $\mathrm{R}^{1}=\mathrm{CH}_{2} \mathrm{CH}_{2} \mathrm{COOCH}_{3}, \mathrm{R}^{2}=\mathrm{H}$

Scheme 2. Synthesis of oximes of 5-formyluracil derivatives.

The oximes $\mathbf{7 a}$ and $\mathbf{7 b}$ were obtained as mixtures of geometrical isomers $E$ and $Z$. Separation of the isomers was performed using column chromatography. The ratio of the isomers strongly depended on the reaction time. After 24 hours only isomer $Z$ of $7 a$ was obtained; when the time of reaction was extended to 6 days the isomer equilibrium settled to a ratio of $Z / E=58: 42$ as determined by ${ }^{1} \mathrm{H} N M R$ analysis. In the case of $7 \mathrm{~b}$ formation of both isomers was observed from the beginning till the end of the reaction: after $24 \mathrm{~h}$ the ratio $Z / E$ was 84:16 and after 6 days changed to $Z / E=26: 74$. Mixtures of stereoisomeric oximes 7a,b were transformed in situ into the corresponding nitrile oxides by a treatment with $N$-chlorosuccinimide (NCS) and triethylamine, these then were directly used in 1,3-dipolar cycloadditions. 
<smiles>[R]n1c(=O)[nH]cc(/C=N/O)c1=O</smiles>

$\mathrm{R}^{1}$

$7 a, b$

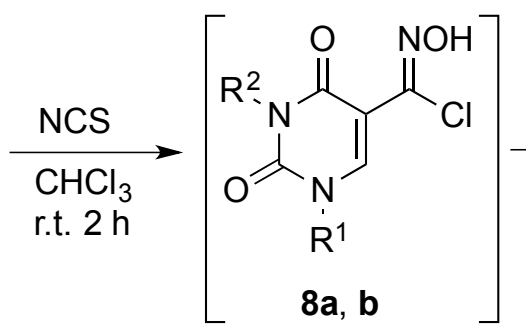

9a $\mathrm{R}^{3}=\mathrm{CN}$

b $\mathrm{R}^{3}=\mathrm{CH}_{2} \mathrm{CN}$

c $\mathrm{R}^{3}=\mathrm{OEt}$

d $\mathrm{R}^{3}=\mathrm{Ph}$<smiles>[R]C1CC(c2c[nH]c(=O)n([R])c2=O)=NO1</smiles>

$\mathrm{R}^{1}$<smiles>[R]C1CON=C1c1c[nH]c(=O)n([R])c1=O</smiles>

$\mathrm{R}^{1}$

$10 \mathrm{~g}$

7, 8, 10 a $\mathrm{R}^{1}, \mathrm{R}^{2}=\mathrm{CH}_{3}, \mathrm{R}^{3}=\mathrm{CN}$

b $\mathrm{R}^{1}=\mathrm{CH}_{2} \mathrm{CH}_{2} \mathrm{COOCH}_{3}, \mathrm{R}^{2}=\mathrm{H}, \mathrm{R}^{3}=\mathrm{CN}$

c $\mathrm{R}^{1}, \mathrm{R}^{2}=\mathrm{CH}_{3}, \mathrm{R}^{3}=\mathrm{CH}_{2} \mathrm{CN}$

d R $\mathrm{R}^{1}=\mathrm{CH}_{2} \mathrm{CH}_{2} \mathrm{COOCH}_{3}, \mathrm{R}^{2}=\mathrm{H}, \mathrm{R}^{3}=\mathrm{CH}_{2} \mathrm{CN}$

e $\mathrm{R}^{1}, \mathrm{R}^{2}=\mathrm{CH}_{3}, \mathrm{R}^{3}=\mathrm{OCH}_{2} \mathrm{CH}_{3}$

$f^{1} R^{1} R^{2}=\mathrm{CH}_{3}, R^{3}=P h$

g R $\mathrm{R}^{1}, \mathrm{R}^{2}=\mathrm{CH}_{3}, \mathrm{R}^{3}=\mathrm{CN}$

Scheme 3. Cycloaddition of nitrile oxides to dipolarophiles $\mathbf{9 a - 9 d . ~}$

To the dipolarophiles (9a-d) we applied unsaturated compounds containing different substituents. In the case of nitrile $9 \mathrm{a}$ the double bond is conjugated with a cyano group but it is isolated in $\mathbf{9 b}$. The cycloaddition occurred in satisfactory yield and in both cases the carbon-carbon double bond participated whereas the nitrile group remained unchanged. We assume that the energy of interacting frontier orbitals of nitrile oxides derived from $\mathbf{8 a}, \mathbf{b}$ correlate better with orbitals of the double bond present in dipolarophiles $\mathbf{9 a -}$ b than with HOMO/LUMO orbitals of nitrile group. In the case of acrylonitrile 9a we observed also traces of a regioisomeric cycloadduct, namely 1,3-dimethyl-5-(4-cyano-4,5-dihydroisoxazol-3-yl)pyrimidine-2,4(1H,3H)dione $10 \mathrm{~g}$ in the post reaction mixture. The obtained cycloadducts are non-aromatic compounds. Among tested oxidizing agents like 2,3-dichloro-5,6-dicyano-1,4-benzoquinone (DDQ), iodine and $N$ bromosuccinimide (NBS) only the latter gave desired 5-(5-substituted-isoxazol-3-yl)pyrimidine-2,4(1H,3H)diones 11 in $40-45 \%$ yield (Scheme 4).<smiles>[R]C1CC(c2cn([R])c(=O)n([R])c2=O)=NO1</smiles>

$10 a, b, g$

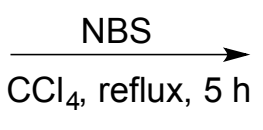

$\mathrm{CCl}_{4}, \mathrm{reflux}, 5 \mathrm{~h}$

$$
\begin{aligned}
& \text { 10-11 a } \mathrm{R}^{1}, \mathrm{R}^{2}=\mathrm{CH}_{3}, \mathrm{R}^{3}=\mathrm{CN} \\
& \text { b } \mathrm{R}^{1}=\mathrm{CH}_{2} \mathrm{CH}_{2} \mathrm{COOCH}_{3}, \mathrm{R}^{2}=\mathrm{H}, \mathrm{R}^{3}=\mathrm{CN} \\
& \text { c } \mathrm{R}^{1}, \mathrm{R}^{2}=\mathrm{CH}_{3}, \mathrm{R}^{3}=\mathrm{Ph}
\end{aligned}
$$

Scheme 4. Aromatization of selected cycloadducts by oxidation with NBS.

For the synthesis of 5-(1,2,4-oxadiazol-3-yl)uracil derivatives we explored a synthetic pathway where uracil derived aldoximes were directly treated, in the presence of cerium ammonium nitrate, with an excess of the appropriate nitrile 12, used as a solvent. ${ }^{58}$ The procedure is simplified in comparison to that described above for the synthesis of 5-(isoxazol-3-yl)uracils. Nevertheless, also in this case, yields of isolated products were only moderate (Scheme 5). 
<smiles>[R]n1cc(C(I)=N[O-])c(=O)n([R])c1=O</smiles>

7a, b

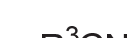

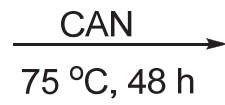
12 a $\mathrm{R}^{3}=\mathrm{CH}_{3}$ b $\mathrm{R}^{3}=\mathrm{Ph}$<smiles>[R]c1nc(-c2c[nH]c(=O)n([R])c2=O)no1</smiles>

$\mathrm{R}^{1}$

7a, 13 a R ${ }^{1}, \mathrm{R}^{2}=\mathrm{CH}_{3}, \mathrm{R}^{3}=\mathrm{CH}_{3}$

7b, 13 b R $\mathrm{R}^{1}=\mathrm{CH}_{2} \mathrm{CH}_{2} \mathrm{COOCH}_{3}, \mathrm{R}^{2}=\mathrm{H}, \mathrm{R}^{3}=\mathrm{CH}_{3}$

$7 \mathrm{a}, 13 c \mathrm{R}^{1}, \mathrm{R}^{2}=\mathrm{CH}_{3}, \mathrm{R}^{3}=\mathrm{Ph}$

Scheme 5. An alternative approach toward the synthesis of 5-hetaryluracils.

The 1,3-dipolar cycloaddition reaction of nitrile oxides to 5-ethynyl uracils has been described in the chemical literature as a method for the preparation of isoxazoles. ${ }^{36}$ We decided to use 5 -cyanouracil 16 , a new commercially available dipolarophile (Scheme 6) for a similar purpose. In this case we generated the nitrile oxide from aromatic aldoximes 14. Application of 5-cyanouracil as a dipolarophile in reactions with aromatic nitrile oxides opened up a new simple route to 5-(1,2,4-oxadiazol-5-yl)uracil derivatives. The cycloaddition reaction was carried out in DMF solution with NCS as the chlorinating agent and triethylamine as a base at room temperature for $24 \mathrm{~h}$. The aldoximes were used in a slight excess in respect to the 5-cyanouracil. Isolation of cycloadducts 17 using column chromatography gave pure products in moderate yields of $44-51 \%$.

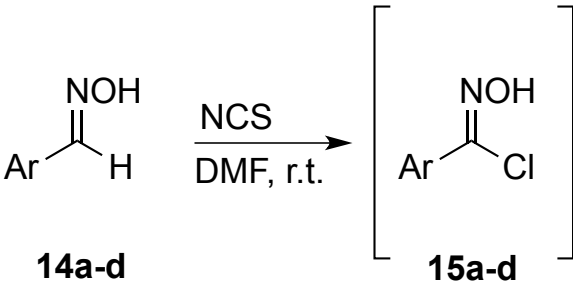

$14 a-d$<smiles>N#Cc1c[nH]c(=O)[nH]c1=O</smiles>

16<smiles>O=c1[nH]cc(-c2nc(Br)no2)c(=O)[nH]1</smiles>

17 a-d

$14,15,17$ a $\mathrm{Ar}=\mathrm{p}-\mathrm{CH}_{3}-\mathrm{C}_{6} \mathrm{H}_{4}-$

b $\mathrm{Ar}=\mathrm{p}-\mathrm{ClC}_{6} \mathrm{H}_{4}-$

c $\mathrm{Ar}=\mathrm{p}-\mathrm{BrC}_{6} \mathrm{H}_{4}^{-}$

d $\mathrm{Ar}=\mathrm{p}-\mathrm{CH}_{3} \mathrm{OC}_{6} \mathrm{H}_{4}-$

Scheme 6. Synthesis of 5-(1,2,4-oxadiazo-5-yl)uracil derivatives.

\section{Conclusions}

We have devised a synthetic pathway for the preparation of various 5-hetaryluracil derivatives using 1,3dipolar cycloaddition reaction. We showed that the studied uracil derivatives could be used either as a source of 1,3-dipoles or dipolarophiles. Nitrile oxides generated in situ from substituted 5-formyluracil oximes treated with alkenes as the dipolarophiles afforded cycloadducts in moderate yields of $40-60 \%$. An oxidation step is necessary to obtain isoxazole derivatives. We also showed that treatment of 5 -formyluracil oximes with nitriles in the presence of ceric ammonium nitrate as an oxidizer leads directly to the respective 5-(oxadiazol3-yl)uracils. 5-Cyanouracil applied as dipolarophile and reacting with aromatic aldoximes gives 5-(oxadiazol-5$\mathrm{yl}$ )uracil derivatives in satisfactory yields. In summary we proved that different types of 5-heteroaryluracil derivatives could be synthesized by manipulation of 1,3-dipole and dipolarophile structures. The cycloadducts 
were obtained in moderate yields regardless of the cycloaddition method applied. Traces of unidentified compounds near to the base line of TLC plates were observed. These are probably the products of nitrile oxide dimerization or isomerisation, like furoxanes or isocyanates etc. In our opinion the moderate yields obtained can be attributed mainly to the crystallization used as a final purification method.

\section{Experimental Section}

General. All reagents and solvents of analytical grade were purchased from commercial suppliers. Most of them were used without further purification except for $\mathrm{CH}_{2} \mathrm{Cl}_{2}$, which was distilled prior to use and for DMF purified by distillation and dried over activated $3 \mathrm{~A}$ molecular sieves. Also anhydrous $\mathrm{Et}_{3} \mathrm{~N}$ was dried through storage over activated 4A molecular sieves. All reactions were monitored by TLC using silica-gel-coated aluminium plates with a fluorescence indicator $\left(\mathrm{SiO}_{2} 60, \mathrm{~F}_{254}\right)$ and spots were visualized by UV light. Column chromatography was performed using silica gel packed columns (particle size 0.040-0.063 mm, Merck). The purified products were obtained as the colorless solids. ${ }^{1} \mathrm{H}$ NMR spectra were recorded at Varian $600 \mathrm{MHz}$ System or Varian Inova $300 \mathrm{MHz}$ spectrometer. ${ }^{13} \mathrm{C}$ NMR spectra were recorded at $150 \mathrm{MHz}$ or $75 \mathrm{MHz}$, respectively. Chemical shifts were measured relative to residual non-deuterated solvent signals. Melting points were determined using a Boethius M HMK hot-stage apparatus. IR spectra by ATR (Attenuated Total Reflection) technique were recorded on Nicolet 6700 FT-IR Spectrometer (Thermo Scientific). High-resolution electrospray ionization mass spectroscopy (ESI-MS) experiments were performed using a Waters Xevo G2 QTOF instrument equipped with an injection system (cone voltage $50 \mathrm{~V}$; source $120{ }^{\circ} \mathrm{C}$ ). 5-Formyl-1,3dimethyluracil (3) was prepared in $72 \%$ yield according to reported method. ${ }^{55}$ 5-(Hydroxymethyl)pyrimidine2,4(1H,3H)-dione $(4)^{56}$ and 2,4-Dioxo-1,2,3,4-tetrahydropyrimidine-5-carbaldehyde $(5)^{56}$ were obtained in yield $95 \%$ and $73 \%$, respectively. The recorded NMR data for 3, 4, and 5 were in accordance with reported data. N1Alkylation of 5-formyluracil using methyl acrylate was performed according to procedure reported earlier. ${ }^{57}$

Methyl 3-(5-formyl-2,4-dioxo-3,4-dihydropyrimidin-1(2H)-yl)propanoate (6). To a suspension of 5-formyl uracil 5 (1.4g, $10 \mathrm{mmol})$ in anhydrous DMF $(25 \mathrm{~mL})$ triethylamine $(1.01 \mathrm{~g}, 1.4 \mathrm{~mL}, 10 \mathrm{mmol})$ was added and the resulting solution was heated in an oil bath at $60{ }^{\circ} \mathrm{C}$ for $0.5 \mathrm{~h}$. Methyl acrylate $(0.56 \mathrm{~mL}, 6 \mathrm{mmol})$ was added dropwise and the mixture was heated for $2 \mathrm{~h}$, then the next portion of methyl acrylate (6 mmol) was added and the reaction was continued for another $2 \mathrm{~h}$. The volatiles were removed at reduced pressure and the residue was crystallized from EtOAc. Yield $1.81 \mathrm{~g}$ (79\%), mp. 153-155 ${ }^{\circ} \mathrm{C}$ (EtOAc). FT IR: $3052,1687,1667,1602$, 1452, $1207 \mathrm{~cm}^{-1} .{ }^{1} \mathrm{H}$ NMR (600 MHz, DMSO-d $)$ ): $\delta 2.76\left(\mathrm{t}, 2 \mathrm{H}, J 6.6, \mathrm{CH}_{2}\right), 3.60\left(\mathrm{~s}, 3 \mathrm{H}, \mathrm{OCH}_{3}\right), 4.04(\mathrm{t}, 2 \mathrm{H}, J 6.6$, $\mathrm{CH}_{2}$ ), 8.47 (s, $\left.1 \mathrm{H}, \mathrm{H}-6\right), 9.77(\mathrm{~s}, 1 \mathrm{H}, \mathrm{CH}=\mathrm{O}), 11.76(\mathrm{~s}, 1 \mathrm{H}, \mathrm{N} 3-\mathrm{H}) .{ }^{13} \mathrm{C} N M R\left(150 \mathrm{MHz}, \mathrm{DMSO}-d_{6}\right): \delta 32.1,45.1$, 51.1, 109.9, 150.0, 152.4, 162.2, 171.2, 186.2. Anal. Calcd for $\mathrm{C}_{9} \mathrm{H}_{10} \mathrm{~N}_{2} \mathrm{O}_{5}$ (226.19): C, 47.97; $\mathrm{H}, 4.46 ; \mathrm{N}, 12.39 \%$. Found: C, 48.21; H, 4. 26; N, 12.16\%.

1,3-Dimethyl-2,4-dioxo-1,2,3,4-tetrahydropyrimidine-5-carbaldehyde oximes (7a). A solution of sodium acetate trihydrate $(0.41 \mathrm{~g}, 3 \mathrm{mmol})$ and $\mathrm{NH}_{2} \mathrm{OH} \cdot \mathrm{HCl}(0.37 \mathrm{~g}, 2.25 \mathrm{mmola})$ in $\mathrm{H}_{2} \mathrm{O}(3 \mathrm{~mL})$ was added to a suspension of uracil $3(0.34 \mathrm{~g}, 2 \mathrm{mmol})$ in EtOH $(5 \mathrm{~mL})$ while stirring at room temperature. After a few minutes a white solid started to precipitate and the stirring was continued for $24 \mathrm{~h}$. A progress of the reaction was monitored by TLC (EtOAc : $n$-hexane, 2:1). The solid consisting of a mixture of two isomeric oximes was isolated in yield of $93 \%(0.34 \mathrm{~g})$. The $\mathrm{E} / \mathrm{Z}$ isomers were separated on silica gel column using $\mathrm{MeOH}^{\mathrm{C}} \mathrm{CHCl}_{3}(5$ : $95, \mathrm{v} / \mathrm{v}$ ) as an eluent. When the time of synthesis was extended to $48 \mathrm{~h} Z$-isomer was obtained exclusively. Z-Isomer: yield 73\% (0.27 g), mp 198-199 ${ }^{\circ} \mathrm{C}(\mathrm{MeOH}) . \mathrm{FT} \mathrm{IR}: 3289,1702,1640,1614,1444,942 \mathrm{~cm}^{-1} .{ }^{1} \mathrm{H} \mathrm{NMR}$ (300 MHz, DMSO- $\left.d_{6}\right): \delta 3.18\left(\mathrm{~s}, 3 \mathrm{H}, \mathrm{N} 1-\mathrm{CH}_{3}\right), 3.38\left(\mathrm{~s}, 3 \mathrm{H}, \mathrm{N} 3-\mathrm{CH}_{3}\right), 7.86(\mathrm{~s}, 1 \mathrm{H}, \mathrm{H}-6), 8.09(\mathrm{~s}, 1 \mathrm{H}, \mathrm{CH}=\mathrm{N}), 11.06(\mathrm{~s}$, 
$1 \mathrm{H}, \mathrm{OH}) .{ }^{13} \mathrm{C}$ NMR $\left(75 \mathrm{MHz}\right.$, DMSO- $\left.d_{6}\right): \delta 27.5,36.7,104.7,141.1,141.4,150.9,161.4$. Anal. Calcd for $\mathrm{C}_{7} \mathrm{H}_{9} \mathrm{~N}_{3} \mathrm{O}_{3}$ (183.16): C, 45.90; H, 4.95; N, 22.94\%. Found: C, 45.56; H, 4.90; N, 22.68\%. E-Isomer: yield $27 \%$ (0.09 g), mp 140-141 ${ }^{\circ} \mathrm{C}$. FT IR: $3287,1700,1643,1610,1442,943 \mathrm{~cm}^{-1} .{ }^{1} \mathrm{H}$ NMR $\left(300 \mathrm{MHz}, \mathrm{DMSO}-d_{6}\right) \delta(\mathrm{ppm}): 3.20$ (s, $3 \mathrm{H}$, $\left.\mathrm{N} 1-\mathrm{CH}_{3}\right), 3.38\left(\mathrm{~s}, 3 \mathrm{H}, \mathrm{N} 3-\mathrm{CH}_{3}\right), 7.34(\mathrm{~s}, 1 \mathrm{H}, \mathrm{H}-6), 8.99(\mathrm{~s}, 1 \mathrm{H}, \mathrm{CH}=\mathrm{N}), 11.83(\mathrm{~s}, 1 \mathrm{H}, \mathrm{OH}) .{ }^{13} \mathrm{C} \mathrm{NMR}(75 \mathrm{MHz}$, DMSO$\left.d_{6}\right) \delta$ (ppm): 27.7, 37.2, 103.2, 136.6, 147.4, 150.4, 161.8. Anal. Calcd for $\mathrm{C}_{7} \mathrm{H}_{9} \mathrm{~N}_{3} \mathrm{O}_{3}$ (183.16): C, 45.90; $\mathrm{H}, 4.95$; N, 22.94\%. Found: C, 45.68; H, 4.89; N, 22.66\%.

Methyl 3-(5-((hydroxyimino)methyl)-2,4-dioxo-3,4-dihydropyrimidin-1(2H)-yl)propanoate (7b). A solution of AcONa*3 $\mathrm{H}_{2} \mathrm{O}(1.63 \mathrm{~g}, 12 \mathrm{mmol})$ and $\mathrm{NH}_{2} \mathrm{OH}^{*} \mathrm{HCl}(0.97 \mathrm{~g}, 14 \mathrm{mmol})$ in $\mathrm{H}_{2} \mathrm{O}(10 \mathrm{~mL})$ was added while stirring to a suspension of 6 (2.29 g, $10 \mathrm{mmol})$ in $\mathrm{EtOH}(20 \mathrm{~mL})$. After a few minutes the suspension disappeared and then white solid started to precipitate from a solution. The stirring was continued for $24 \mathrm{~h}$. The solid was filtered off and purified on silica gel column using a mixture of $\mathrm{CHCl}_{3}: \mathrm{MeOH}(95: 5, \mathrm{v} / \mathrm{v})$ as an eluent. The separated isomers were crystallized from MeOH. Z-Isomer: yield 84\% (1.81 g), mp 179-180 ${ }^{\circ} \mathrm{C}$ (MeOH). FT IR: 3296, 1679, $1467,1208,948 \mathrm{~cm}^{-1} .{ }^{1} \mathrm{H}$ NMR $\left(600 \mathrm{MHz}, \mathrm{DMSO}-d_{6}\right) \delta(\mathrm{ppm}): 2.73\left(\mathrm{t}, 2 \mathrm{H}, J 6.6, \mathrm{CH}_{2}\right), 3.60\left(\mathrm{~s}, 3 \mathrm{H}, \mathrm{OCH}_{3}\right), 3.97(\mathrm{t}$, $2 \mathrm{H}$, J 6.6, $\left.\mathrm{CH}_{2}\right), 7.81(\mathrm{~s}, 1 \mathrm{H}, \mathrm{H}-6), 8.05(\mathrm{~s}, 1 \mathrm{H}, \mathrm{CH}=\mathrm{N}), 11.07(\mathrm{~s}, 1 \mathrm{H}, \mathrm{OH}), 11.57(\mathrm{~s}, 1 \mathrm{H}, \mathrm{N} 3-\mathrm{H}) .{ }^{13} \mathrm{C} \mathrm{NMR}(150 \mathrm{MHz}$, DMSO-d $\left.d_{6}\right) \delta(\mathrm{ppm}): 32.4,44.5,51.5,105.7,140.9,142.4,150.2,162.1,171.3$. Anal. Calcd for $\mathrm{C}_{9} \mathrm{H}_{11} \mathrm{~N}_{3} \mathrm{O}_{5}$ (241.20): C, 44.82; H, 4.60; N, 17.42\%. Found: C, 44.58; H, 4.83; N, 17.38\%. E-Isomer: yield 16\% (0.34 g), mp $189-190{ }^{\circ} \mathrm{C}(\mathrm{MeOH}) . \mathrm{FT} \mathrm{IR:} 3295,1677,1464,1206,945 \mathrm{~cm}^{-1} .{ }^{1} \mathrm{H}$ NMR $\left(600 \mathrm{MHz}, \mathrm{DMSO}-d_{6}\right) \delta(\mathrm{ppm}): 2.77-2.68$ $\left(\mathrm{m}, 2 \mathrm{H}, \mathrm{CH}_{2}\right), 3.59\left(\mathrm{~s}, 3 \mathrm{H}, \mathrm{OCH}_{3}\right), 4.01-3.91\left(\mathrm{~m}, 2 \mathrm{H}, \mathrm{CH}_{2}\right), 7.28(\mathrm{~s}, 1 \mathrm{H}, \mathrm{H}-6), 9.03(\mathrm{~s}, 1 \mathrm{H}, \mathrm{CH}=\mathrm{N}), 11.64(\mathrm{~s}, 1 \mathrm{H}, \mathrm{OH})$, 11.82 (s, $1 \mathrm{H}, \mathrm{N} 3-\mathrm{H}) .{ }^{13} \mathrm{C}$ NMR (150 MHz, DMSO-d 6 ) $\delta$ (ppm): 32.5, 45.1, 51.6, 104.1, 136.2, 149.1, 149.7, 162.6, 171.2. Anal. Calcd for $\mathrm{C}_{9} \mathrm{H}_{11} \mathrm{~N}_{3} \mathrm{O}_{5}$ (241.20): C, 44.82; $\mathrm{H}, 4.60 ; \mathrm{N}, 17.42 \%$. Found: $\mathrm{C}, 44.60 ; \mathrm{H}, 4.58 ; \mathrm{N}, 17.28 \%$.

General procedure for preparation of 5-(4,5-Dihydroisoxazol-3-yl)-1,3-dimethylpyrimidine-2,4(1H,3H)diones (10a-f).To a suspension of oxime 7 a or $7 \mathrm{~b}(0.77 \mathrm{mmol})$ in $\mathrm{CHCl}_{3}$ or $\mathrm{DMF}(3 \mathrm{~mL}) \mathrm{N}$-chlorosuccinimide $(0.11 \mathrm{~g}, 0.85 \mathrm{mmol})$ and concentrated hydrochloric acid (1 drop) was added while stirring. An appropriate dipolarophile $9 \mathrm{a}-\mathbf{d}(0.7 \mathrm{mmol})$ and triethylamine $(0.11 \mathrm{~mL}, 0.77 \mathrm{mmol})$ were added after $2 \mathrm{~h}$. The stirring was continued for $24 \mathrm{~h}$. The volatiles were removed under reduced pressure and the residue was purified on silica gel column (EtOAc: $n$-hexane, 3:1, v/v).

3-(1,3-Dimethyl-2,4-dioxo-1,2,3,4-tetrahydropyrimidin-5-yl)-4,5-dihydroisoxazole-5-carbonitrile (10a). Yield

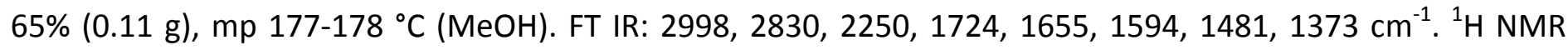
(300 MHz, DMSO-d $)_{6} \delta(\mathrm{ppm}): 3.20\left(\mathrm{~s}, 3 \mathrm{H}, \mathrm{N} 1-\mathrm{CH}_{3}\right), 3.39\left(\mathrm{~s}, 3 \mathrm{H}, \mathrm{N} 3-\mathrm{CH}_{3}\right), 3.80-3.73\left(\mathrm{~m}, 2 \mathrm{H}, \mathrm{H}_{\mathrm{A}}-4^{\prime}, \mathrm{H}_{\mathrm{B}}-4^{\prime}\right)$, $5.73(\mathrm{dd}, 1 \mathrm{H}, J$ 9.0, 6.6, H-5') 8.35 (s, $1 \mathrm{H}, \mathrm{H}-6) .{ }^{13} \mathrm{C} \mathrm{NMR}\left(75 \mathrm{MHz}, \mathrm{DMSO}-d_{6}\right) \delta(\mathrm{ppm}): 27.7\left(\mathrm{~N} 1-\mathrm{CH}_{3}\right), 36.9(\mathrm{~N} 3-$ $\mathrm{CH}_{3}$ ), 41.9 (C-4'), 66.3 (C-5'), 100.4 (C-5), 118.5 (CN), 145.5 (C-6), 150.7 (C-2), 153.3 (C-3'), 160.2 (C-4). Anal. Calcd for $\mathrm{C}_{10} \mathrm{H}_{10} \mathrm{~N}_{4} \mathrm{O}_{3}$ (234.21): C, 51.28; $\mathrm{H}, 4.30 ; \mathrm{N}, 23.92 \%$. Found: $\mathrm{C}, 51.08 ; \mathrm{H}, 4.13 ; \mathrm{N}, 23.68 \%$.

Methyl 3-(5-(5-cyano-4,5-dihydroisoxazol-3-yl)-2,4-dioxo-3,4-dihydropyrimidin-1(2H)-yl)propanoate (10b). Yield 56\% (0.12 g), mp 162-165 ${ }^{\circ} \mathrm{C}(\mathrm{MeOH}) . \mathrm{FT} \mathrm{IR:} \mathrm{3166,} \mathrm{3049,} \mathrm{2823,} \mathrm{2250,} \mathrm{1737,} \mathrm{1683,} 1464 \mathrm{~cm}^{-1} .{ }^{1} \mathrm{H}$ NMR (300 $\left.\mathrm{MHz}, \mathrm{DMSO}-d_{6}\right) \delta(\mathrm{ppm}): 2.75\left(\mathrm{t}, 2 \mathrm{H}, J 6.4, \mathrm{CH}_{2} \mathrm{COOCH}_{3}\right), 3.61\left(\mathrm{~s}, 3 \mathrm{H}, \mathrm{OCH}_{3}\right), 11.71(\mathrm{~s}, 1 \mathrm{H}, \mathrm{N} 3-\mathrm{H}), 3.74(\mathrm{~d}, 2 \mathrm{H}, J$ 7.8, $\left.\mathrm{H}_{\mathrm{A}}-4^{\prime}, \mathrm{H}_{\mathrm{B}}-4^{\prime}\right), 3.99\left(\mathrm{t}, 2 \mathrm{H}, \mathrm{J} 6.4, \mathrm{~N} 1-\mathrm{CH}_{2}\right), 5.70\left(\mathrm{t}, 1 \mathrm{H}, \mathrm{J} 7.8, \mathrm{H}-5^{\prime}\right), 8.29(\mathrm{~s}, 1 \mathrm{H}, \mathrm{H}-6) .{ }^{13} \mathrm{C} \mathrm{NMR}(75 \mathrm{MHz}$, DMSO$\left.d_{6}\right) \delta(\mathrm{ppm}): 32.3\left(\mathrm{CH}_{2} \mathrm{COOCH}_{3}\right), 41.9\left(\mathrm{C}-4^{\prime}\right), 44.5\left(\mathrm{~N}-\mathrm{CH}_{2}\right), 51.6\left(\mathrm{OCH}_{3}\right), 66.2\left(\mathrm{C}-5^{\prime}\right), 101.4(\mathrm{C}-5), 118.5(\mathrm{CN})$, 147.0 (C-6), 150.0 (C-2), 153.0 (C-3'), 161.0 (C-4), $171.2\left(\mathrm{COOCH}_{3}\right)$. Anal. Calcd for $\mathrm{C}_{12} \mathrm{H}_{12} \mathrm{~N}_{4} \mathrm{O}_{5}$ (292.25): C, 49.32; H, 4.14; N, 19.17\%. Found: C, 49.58; H, 4.03; N, 18.95\%.

2-(3-(1,3-Dimethyl-2,4-dioxo-1,2,3,4-tetrahydropyrimidin-5-yl)-4,5-dihydroisoxazol-5-yl)acetonitrile (10c). Yield 35\% (0.06 g), mp 206-208 ${ }^{\circ} \mathrm{C}$ (EtOH). FT IR: 3059, 2950, 2256, 1694, 1640, 1449, $1343 \mathrm{~cm}^{-1} .{ }^{1} \mathrm{H}$ NMR (300 $\left.\mathrm{MHz}, \mathrm{DMSO}-d_{6}\right) \delta(\mathrm{ppm}): 2.00-2.80\left(\mathrm{~m}, 2 \mathrm{H}, \mathrm{CH}_{2} \mathrm{CN}\right), 3.24-3.13\left(\mathrm{~m}, 4 \mathrm{H}, \mathrm{H}_{\mathrm{B}}-4^{\prime}, \mathrm{N} 1-\mathrm{CH}_{3}\right), 3.39\left(\mathrm{~s}, 3 \mathrm{H}, \mathrm{N} 3-\mathrm{CH}_{3}\right), 3.54$ (dd, $\left.1 \mathrm{H}, J 18.0,10.5, \mathrm{H}_{\mathrm{A}}-4^{\prime}\right)$, 4.94-4.82 (m, $\left.1 \mathrm{H}, \mathrm{H}-5^{\prime}\right), 8.25$ (s, $\left.1 \mathrm{H}, \mathrm{H}-6\right) .{ }^{13} \mathrm{C} \mathrm{NMR}\left(75 \mathrm{MHz}, \mathrm{DMSO}-d_{6}\right) \delta(\mathrm{ppm})$ : 
$22.7\left(\mathrm{CH}_{2} \mathrm{CN}\right), 27.6\left(\mathrm{~N} 1-\mathrm{CH}_{3}\right), 36.8\left(\mathrm{N3}^{-} \mathrm{CH}_{3}\right), 41.0$ (C-4'), 75.0 (C-5'), 101.6 (C-5), 117.9 (CN), 144.3 (C-6), 150.8 (C-2), 152.7 (C-3'), 160.4 (C-4). ESI-MS [M+H] calcd. for $\mathrm{C}_{11} \mathrm{H}_{13} \mathrm{~N}_{4} \mathrm{O}_{3}$ (249.0989). Found 249.1011. Anal. Calcd for $\mathrm{C}_{11} \mathrm{H}_{12} \mathrm{~N}_{4} \mathrm{O}_{3}$ (248.24): $\mathrm{C}, 53.22 ; \mathrm{H}, 4.87 ; \mathrm{N}, 22.57 \%$. Found: $\mathrm{C}, 53.08 ; \mathrm{H}, 4.63 ; \mathrm{N}, 22.35 \%$.

Methyl 3-(5-(5-(cyanomethyl)-4,5-dihydroisoxazol-3-yl)-2,4-dioxo-3,4-dihydropyrimidin-1(2H)-yl)propanoate (10d). Yield 38\% (0.08 g), mp 181-183 ${ }^{\circ} \mathrm{C}$ (EtOH). FT IR: 3172, 3051, 2250, 1735, 1708, 1651, 1457, $1204 \mathrm{~cm}^{-1}$. ${ }^{1} \mathrm{H}$ NMR $\left(300 \mathrm{MHz}, \mathrm{DMSO}-d_{6}\right) \delta(\mathrm{ppm}): 2.74\left(\mathrm{t}, 2 \mathrm{H}, \mathrm{J} 6.6, \mathrm{CH}_{2} \mathrm{COOCH}_{3}\right), 2.99-2.81\left(\mathrm{~m}, 2 \mathrm{H}, \mathrm{CH}_{2} \mathrm{CN}\right), 3.17(\mathrm{dd}, 1 \mathrm{H}, J$ 17.7, 6.9, $\left.\mathrm{H}_{\mathrm{B}}-4^{\prime}\right), 3.51\left(\mathrm{dd}, 1 \mathrm{H}, J 17.7,10.2, \mathrm{H}_{\mathrm{A}}-4^{\prime}\right), 3.61\left(\mathrm{~s}, 3 \mathrm{H}, \mathrm{OCH}_{3}\right), 3.99\left(\mathrm{t}, 2 \mathrm{H}, J 6.6, \mathrm{~N} 1-\mathrm{CH}_{2}\right), 4.93-4.80(\mathrm{~m}$, $\left.1 \mathrm{H}, \mathrm{H}-5^{\prime}\right), 8.19(\mathrm{~s}, 1 \mathrm{H}, \mathrm{H}-6), 11.76(\mathrm{~s}, 1 \mathrm{H}, \mathrm{N} 3-\mathrm{H}) .{ }^{13} \mathrm{C} \mathrm{NMR}\left(75 \mathrm{MHz}, \mathrm{DMSO}-d_{6}\right) \delta(\mathrm{ppm}): 23.7\left(\mathrm{CH}_{2} \mathrm{CN}\right), 32.4$ $\left(\mathrm{CH}_{2} \mathrm{COOCH}_{3}\right), 41.0\left(\mathrm{C}-4^{\prime}\right), 44.5\left(\mathrm{~N} 1-\mathrm{CH}_{2}\right), 51.6\left(\mathrm{OCH}_{3}\right), 75.0\left(\mathrm{C}-5^{\prime}\right), 102.7(\mathrm{C}-5), 117.9(\mathrm{CN}), 145.8(\mathrm{C}-6), 150.1(\mathrm{C}-$ 2), 152.3 (C-3'), $161.2(\mathrm{C}-4), 171.4\left(\mathrm{COOCH}_{3}\right)$. ESI-MS [M+H] ${ }^{+}$calcd. for $\mathrm{C}_{13} \mathrm{H}_{15} \mathrm{~N}_{4} \mathrm{O}_{5}$ (307.1043). Found 307.0998. 5-(5-Ethoxy-4,5-dihydroisoxazol-3-yl)-1,3-dimethylpyrimidine-2,4(1H,3H)-dione (10e). Yield 47\% (0.08 g), mp 177-179 ${ }^{\circ} \mathrm{C}$ (EtOH). FT IR: 3067, 2977, 1647, 1457, $1345 \mathrm{~cm}^{-1} .{ }^{1} \mathrm{H}$ NMR $\left(600 \mathrm{MHz}, \mathrm{CDCl}_{3}\right) \delta$ (ppm): 3.30 (dd, $1 \mathrm{H}, J$ 18.0, $\left.1.8 \mathrm{~Hz}, \mathrm{H}_{\mathrm{B}}-4^{\prime}\right), 3.37\left(\mathrm{~s}, 3 \mathrm{H}, \mathrm{N} 1-\mathrm{CH}_{3}\right), 3.47\left(\mathrm{~s}, 3 \mathrm{H}, \mathrm{N} 3-\mathrm{CH}_{3}\right), 3.56\left(\mathrm{dd}, 1 \mathrm{H}, J 18.0,7.2, \mathrm{H}_{\mathrm{A}}-4^{\prime}\right), 3.58(\mathrm{dq}, 1 \mathrm{H}, J$ 9.6, 7.2, $\mathrm{OCH}_{2 B} \mathrm{CH}_{3}$ ), 3.86 (dq, $1 \mathrm{H}, J$ 9.6, $7.2 \mathrm{~Hz}, \mathrm{OCH}_{2 \mathrm{~A}} \mathrm{CH}_{3}$ ), 5.61 (dd, $1 \mathrm{H}, J$ 7.2, 1.8, H-5'), $7.96(\mathrm{~s}, 1 \mathrm{H}, \mathrm{H}-6) .{ }^{13} \mathrm{C}$ NMR $\left(150 \mathrm{MHz}, \mathrm{CDCl}_{3}\right) \delta(\mathrm{ppm}): 15.0\left(\mathrm{OCH}_{2} \underline{\mathrm{CH}}_{3}\right), 28.1\left(\mathrm{~N} 1-\mathrm{CH}_{3}\right), 37.5\left(\mathrm{~N} 3-\mathrm{CH}_{3}\right), 43.1\left(\mathrm{C}-4^{\prime}\right), 63.8,\left(\mathrm{OCH}_{2} \mathrm{CH}_{3}\right)$, 103.2 (C-5'), 103.6 (C-5), 145.9 (C-6), 151.2 (C-2), 153.1 (C-3'), 161.1 (C-4). Anal. Calcd for $\mathrm{C}_{11} \mathrm{H}_{15} \mathrm{~N}_{3} \mathrm{O}_{4}(253.25)$ : C, 52.17; H, 5.97; N, 16.59\%. Found: C, 51.94; H, 5.67; N, 16.36\%.

1,3-Dimethyl-5-(5-phenyl-4,5-dihydroisoxazol-3-yl)pyrimidine-2,4(1H,3H)-dione (10f). Yield 60\% (0.14 g), mp 150-152 ${ }^{\circ} \mathrm{C}$ (MeOH). FT IR: 3061, 2952, 1698, 1648, 1447, $1344 \mathrm{~cm}^{-1} .{ }^{1} \mathrm{H}$ NMR $\left(600 \mathrm{MHz}, \mathrm{CDCl}_{3}\right) \delta(\mathrm{ppm}): 3.36$

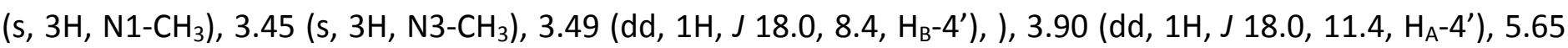
(dd, $1 \mathrm{H}, J$ J 11.4, 8.4, H-5'), 7.33-7.28 (m, 1H, Ar-H), 7.39-7.34 (m, 4H, ArH), $7.95(\mathrm{~s}, 1 \mathrm{H}, \mathrm{H}-6) .{ }^{13} \mathrm{C} \mathrm{NMR}(150 \mathrm{MHz}$, $\left.\mathrm{CDCl}_{3}\right) \delta(\mathrm{ppm}): 28.1\left(\mathrm{~N} 1-\mathrm{CH}_{3}\right), 37.5\left(\mathrm{~N}_{-}-\mathrm{CH}_{3}\right), 44.1$ (C-4'), 82.7 (C-5'), 103.8 (C-5), 125.9 (2 x C-Ph), 128.2 (C-4$\mathrm{Ph}), 128.7$ (2 x C-Ph), 140.6 (C-1-Ph), 142.2 (C-6), 151.2 (C-2), 152.5 (C-3'), 162.2 (C-4). ESI-MS [M+H] ${ }^{+}$Calcd for $\mathrm{C}_{15} \mathrm{H}_{16} \mathrm{~N}_{3} \mathrm{O}_{3}$ 286.1192. Found 286.1675.

3-(1,3-Dimethyl-2,4-dioxo-1,2,3,4-tetrahydropyrimidin-5-yl)-4,5-dihydroisoxazole-4-carbonitrile (10g). Yield $2 \%(0.003 \mathrm{~g}), \mathrm{mp} 175-178{ }^{\circ} \mathrm{C}$. FT IR: 2996, 2248, 1723, 1652, 1592, 1480, $1371 \mathrm{~cm}^{-1} .{ }^{1} \mathrm{H} \mathrm{NMR}\left(300 \mathrm{MHz}_{1} \mathrm{CDCl}_{3}\right)$ $\delta(\mathrm{ppm}): 3.41\left(\mathrm{~s}, 3 \mathrm{H}, \mathrm{N} 1-\mathrm{CH}_{3}\right), 3.52\left(\mathrm{~s}, 3 \mathrm{H}, \mathrm{N} 3-\mathrm{CH}_{3}\right), 4.62\left(\mathrm{dd}, 1 \mathrm{H}, J 11.1,8.7, \mathrm{H}_{\mathrm{A}}-5^{\prime}\right), 4.71(\mathrm{dd}, 1 \mathrm{H}, J$ 8.7, 7.2, H$\left.4^{\prime}\right), 5.73\left(\mathrm{dd}, 1 \mathrm{H}, J 11.1,7.2 \mathrm{~Hz}, \mathrm{H}_{\mathrm{B}}-5^{\prime}\right), 7.97$ (s, $\left.1 \mathrm{H}, \mathrm{H}-6\right) .{ }^{13} \mathrm{C} \mathrm{NMR}\left(75 \mathrm{MHz}, \mathrm{CDCl}_{3}\right.$ ) $\delta(\mathrm{ppm}): 28.4,37.9,39.5$, 72.7, 100.7, 116.0, 143.9, 148.2, 150.9, 160.0. ESI-MS $[\mathrm{M}+\mathrm{H}]^{+}$Calcd for $\mathrm{C}_{10} \mathrm{H}_{11} \mathrm{~N}_{4} \mathrm{O}_{3}$ (235.2193). Found 235.2084 .

General procedure for oxidation of cycloadducts (10a-f). To the 4,5-dihydroisoxazole derivative $\mathbf{1 0 a}-\mathbf{f}(0.5$ $\mathrm{mmol})$ in $\mathrm{CCl}_{4}(4 \mathrm{~mL}) \mathrm{NBS}(0.13 \mathrm{~g}, 0.74 \mathrm{mmol})$ was added and the reaction mixture was refluxed and monitored by TLC ( $\mathrm{MeOH}: \mathrm{CHCl}_{3}, 5: 95, \mathrm{v} / \mathrm{v}$ ). After disappearance of 4,5-dihydroisoxazole (usually 4-5 h) the reaction mixture was evaporated to dryness and the residue was purified on silica gel column using a mixture of $\mathrm{MeOH}: \mathrm{CHCl}_{3}(5: 95, \mathrm{v} / \mathrm{v})$ as an eluent.

3-(1,3-Dimethyl-2,4-dioxo-1,2,3,4-tetrahydropyrimidin-5-yl)isoxazole-5-carbonitrile (11a). Yield 45\% (0.05 g), $\mathrm{mp} 187-189^{\circ} \mathrm{C}$ (MeOH). FT IR: 3186, 3069, 2246, 1702, 1645, 1479, $1350 \mathrm{~cm}^{-1} .{ }^{1} \mathrm{H}$ NMR $\left(300 \mathrm{MHz}, \mathrm{DMSO}-d_{6}\right) \delta$ (ppm): 3.43 (s, 3H, N1-CH $), 3.55\left(\mathrm{~s}, 3 \mathrm{H}, \mathrm{N} 3-\mathrm{CH}_{3}\right), 7.74\left(\mathrm{~s}, 1 \mathrm{H}, \mathrm{H}-4{ }^{\prime}\right), 8.19(\mathrm{~s}, 1 \mathrm{H}, \mathrm{H}-6) .{ }^{13} \mathrm{C} \mathrm{NMR}(75 \mathrm{MHz}$, DMSO$\left.d_{6}\right) \delta$ (ppm): 28.4, 37.9, 96.3, 100.8, 108.3, 113.9, 142.7, 143.1, 157.3, 160.9. Anal. Calcd for $\mathrm{C}_{10} \mathrm{H}_{8} \mathrm{~N}_{4} \mathrm{O}_{3}$ (232.20): C, 51.73, H, 3.47, N, 24.13\%. Found: C, 51.49, H, 3.28; N, 24.05\%.

Methyl 3-(5-(5-cyanoisoxazol-3-yl)-2,4-dioxo-3,4-dihydropyrimidin-1(2H)-yl)propanoate (11b). Yield 43\% (0.06 g), mp 182-185 ${ }^{\circ} \mathrm{C}(\mathrm{MeOH})$. FT IR: 3180, 2246, 1700, 1650, $1470 \mathrm{~cm}^{-1} .{ }^{1} \mathrm{H}$ NMR (300 MHz, DMSO-d $d_{6} \delta$ (ppm): $2.77\left(\mathrm{t}, 2 \mathrm{H}, J\right.$ 6.6, N1- $\left.\mathrm{CH}_{2}\right), 3.60\left(\mathrm{~s}, 3 \mathrm{H}, \mathrm{OCH}_{3}\right), 4.04\left(\mathrm{t}, 2 \mathrm{H}, J\right.$ 6.6, $\left.\mathrm{CH}_{2} \mathrm{COOCH}_{3}\right), 7.96\left(\mathrm{~s}, 1 \mathrm{H}, \mathrm{H}-4^{\prime}\right), 8.54(\mathrm{~s}$, 
$1 \mathrm{H}, \mathrm{H}-6), 11.87$ (s, $1 \mathrm{H}, \mathrm{N} 3-\mathrm{H}) .{ }^{13} \mathrm{C}$ NMR (75 MHz, DMSO-d $) \delta$ (ppm): 32.2, 44.6, 51.5, 79.1, 99.5, 114.6, 141.4, 146.8, 150.0, 157.3, 161.1, 171.1. Anal. Calcd for $\mathrm{C}_{12} \mathrm{H}_{10} \mathrm{~N}_{4} \mathrm{O}_{5}$ (290.23): C, 49.66, H, 3.47, N, 19.30\%. Found: C, 49.52, H, 3.27, N, 19.05\%.

1,3-Dimethyl-5-(5-phenylisoxazol-3-yl)pyrimidine-2,4(1H,3H)-dione (11c). Yield 40\% (0.06 g), mp $226-227{ }^{\circ} \mathrm{C}$ (EtOH). FT IR: 3061, 1699, 1674, 1609, 1515, $1452 \mathrm{~cm}^{-1} .{ }^{1} \mathrm{H}$ NMR $\left(600 \mathrm{MHz}, \mathrm{CDCl}_{3}\right) \delta$ (ppm): 3.27 (s, 3H, N1$\left.\mathrm{CH}_{3}\right), 3.39\left(\mathrm{~s}, 3 \mathrm{H}, \mathrm{N} 3-\mathrm{CH}_{3}\right), 7.37\left(\mathrm{~s}, 1 \mathrm{H}, \mathrm{H}-4^{\prime}\right), 7.59-7.50(\mathrm{~m}, 3 \mathrm{H}, \mathrm{ArH}), 7.90(\mathrm{~d}, 2 \mathrm{H}, J 7.2 \mathrm{ArH}), 8.45(\mathrm{~s}, 1 \mathrm{H}, \mathrm{H}-6)$. ${ }^{13} \mathrm{C} \mathrm{NMR}\left(150 \mathrm{MHz}, \mathrm{CDCl}_{3}\right) \delta(\mathrm{ppm}): 27.8\left(\mathrm{~N} 1-\mathrm{CH}_{3}\right), 36.9\left(\mathrm{~N} 3-\mathrm{CH}_{3}\right), 100.2\left(\mathrm{C}-4^{\prime}\right), 100.6(\mathrm{C}-5), 125.6(2 \times \mathrm{C}-\mathrm{Ph})$, 126.8 (C-1-Ph), 129.3 (2 x C-Ph), 130.4 (C-4-Ph), 144.4 (C-6), 150.9 (C-2), 157.5 (C-3'), 160.7 (C-4), 168.7 (C-5'). Anal. Calcd for $\mathrm{C}_{15} \mathrm{H}_{13} \mathrm{~N}_{3} \mathrm{O}_{3}$ (283.28): $\mathrm{C}, 63.60 ; \mathrm{H}, 4.63 ; \mathrm{N}, 14.83 \%$. Found: $\mathrm{C}, 63.48 ; \mathrm{H}, 4.38 ; \mathrm{N}, 14.66 \%$.

General procedure for cycloaddition of $7 \mathrm{a}$ and $7 \mathrm{~b}$ to nitriles in the presence of CAN. CAN $(0.33 \mathrm{~g}, 0.6 \mathrm{mmol})$ was added to a suspension of oxime $7 \mathbf{a}$ or $7 \mathbf{b}(0.6 \mathrm{mmol})$ in acetonitrile $(16 \mathrm{~mL})$ and the resulting suspension was stirred in an oil bath at $75{ }^{\circ} \mathrm{C}$ for $48 \mathrm{~h}$. After spot of substrate decay ( $\left.\mathrm{TLC} \mathrm{MeOH:CHCl}, 1: 9\right)$, the solvent was evaporated and the residue was purified on silica gel column (MeOH: $\mathrm{CHCl}_{3}, 5: 95$ or 1:9, v/v).

1,3-Dimethyl-5-(5-methyl-1,2,4-oxadiazol-3-yl)pyrimidine-2,4(1H,3H)-dione (13a). Yield 47\% (0.07 g), mp 230-232 ${ }^{\circ} \mathrm{C}$ (MeOH). FT IR: 3040, 1680, 1467, $1270 \mathrm{~cm}^{-1} .{ }^{1} \mathrm{H}$ NMR (300 MHz, DMSO-d 6 ) $\delta(p p m): 2.62(\mathrm{~s}, 3 \mathrm{H}$, $\left.\mathrm{C}^{\prime}-\mathrm{CH}_{3}\right), 3.44\left(\mathrm{~s}, 3 \mathrm{H}, \mathrm{N} 1-\mathrm{CH}_{3}\right), 3.54\left(\mathrm{~s}, 3 \mathrm{H}, \mathrm{N} 3-\mathrm{CH}_{3}\right), 8.17$ (s, $\left.1 \mathrm{H}, \mathrm{H}-6\right) .{ }^{13} \mathrm{C}$ NMR $\left(75 \mathrm{MHz}, \mathrm{DMSO}-d_{6}\right) \delta(\mathrm{ppm})$ : 12.2, 28.2, 37.6, 102.1, 145.1, 151.0, 159.3, 163.4, 175.6. Anal. Calcd for $\mathrm{C}_{9} \mathrm{H}_{10} \mathrm{~N}_{4} \mathrm{O}_{3}$ (222.20): C, 48.65; $\mathrm{H}, 4.54$; N, 25.21\%. Found: C, 48.54; H, 4.27; N, 24.98\%.

Methyl 3-(5-(5-methyl-1,2,4-oxadiazol-3-yl)-2,-dioxo-3,4-dihydropyrimidin-1(2H)-yl)propanoate (13b). Yield $44 \%$ (0.08 g), mp 189-192 ${ }^{\circ} \mathrm{C}$ (EtOH). FT IR: 3038, 1683, 1463, $1261 \mathrm{~cm}^{-1} .{ }^{1} \mathrm{H}$ NMR $\left(300 \mathrm{MHz}, \mathrm{DMSO}-d_{6}\right) \delta(\mathrm{ppm}):$ $2.61\left(\mathrm{~s}, 3 \mathrm{H}, \mathrm{C5}^{\prime}-\mathrm{CH}_{3}\right), 2.76\left(\mathrm{t}, 2 \mathrm{H}, J\right.$ 6.6, $\left.\mathrm{NCH}_{2}\right), 3.60\left(\mathrm{~s}, 3 \mathrm{H}, \mathrm{OCH}_{3}\right), 4.06\left(\mathrm{t}, 2 \mathrm{H}, \mathrm{J}=6.6, \mathrm{CH}_{2} \mathrm{COOCH}_{3}\right), 8.48(\mathrm{~s}, 1 \mathrm{H}$, $\mathrm{H}-6), 11.71$ (s, $1 \mathrm{H}, \mathrm{N} 3-\mathrm{H}) .{ }^{13} \mathrm{C}$ NMR $\left(75 \mathrm{MHz}, \mathrm{DMSO}-d_{6}\right) \delta(\mathrm{ppm}): 11.6,32.2,44.7,51.5,100.8,148.8,149.9$, 159.6, 163.0, 171.2, 175.5. Anal. Calcd for $\mathrm{C}_{11} \mathrm{H}_{12} \mathrm{~N}_{4} \mathrm{O}_{5}$ (280.24): C, 47.15; H, 4.32; N, 19.99\%. Found: C, 47.24; H, 4.15; N, 19.78\%.

1,3-Dimethyl-5-(5-phenyl-1,2,4-oxadiazol-3-yl)pyrimidine-2,4(1H,3H)-dione (13c). Oxime 7a (0.6 mmol) and CAN $(0.33 \mathrm{~g}, 0.6 \mathrm{mmol})$ were heated in benzonitrile $(8 \mathrm{~mL})$ at $75{ }^{\circ} \mathrm{C}$ for $48 \mathrm{~h}$. The workup as above gave the product. Yield 38\% (0.07 g), mp 207-208 ${ }^{\circ} \mathrm{C}(\mathrm{MeOH})$. FT IR: 3063, 1710, 1657, 1636, 1452, $1327 \mathrm{~cm}^{-1} .{ }^{1} \mathrm{H}$ NMR $\left(300 \mathrm{MHz}, \mathrm{CDCl}_{3}\right) \delta(\mathrm{ppm}): 3.45\left(\mathrm{~s}, 3 \mathrm{H}, \mathrm{N} 1-\mathrm{CH}_{3}\right), 3.57\left(\mathrm{~s}, 3 \mathrm{H}, \mathrm{N} 3-\mathrm{CH}_{3}\right), 7.53(\mathrm{t}, 2 \mathrm{H}, J$ 7.2, ArH), $7.61(\mathrm{t}, 1 \mathrm{H}, J 7.2$, $\mathrm{ArH}), 8.16(\mathrm{~d}, 2 \mathrm{H}, J \mathrm{7.2}, \mathrm{ArH}), 8.31(\mathrm{~s}, 1 \mathrm{H}, \mathrm{H}-6) .{ }^{13} \mathrm{C} \mathrm{NMR}\left(75 \mathrm{MHz}, \mathrm{CDCl}_{3}\right) \delta(\mathrm{ppm}): 28.3,37.7,102.2,123.8$, $128.1,129.1,132.9,145.5,151.0,159.3,163.8,172.3$, Anal. Calcd for $\mathrm{C}_{14} \mathrm{H}_{12} \mathrm{~N}_{4} \mathrm{O}_{3}$ (284.27): C, 59.15; $\mathrm{H}, 4.25 ; \mathrm{N}$, 19.71\%. Found: C, 58.89; H, 4.19; N, 19.58\%.

General procedure for preparation of uracils (17a-d). NCS $(0.06 \mathrm{~g}, 0.44 \mathrm{mmol})$ was added while stirring to a solution of oxime $14 a-d^{55}(0.4 \mathrm{mmol})$ in dry DMF $(3 \mathrm{~mL})$ at room temperature. The completion of the reaction was indicated by TLC (EtOAc : $n$-hexane, $1: 1 \mathrm{v} / \mathrm{v}$ ). The solution of generated oxymoyl chloride 15a-d was immediately used for the next step without purification. 5-Cyanouracil 16 (0.05 g, $0.35 \mathrm{mmol})$ was added followed by dropwise addition of triethylamine $(0.06 \mathrm{~mL}, 0.4 \mathrm{mmol})$. The reaction mixture was stirred for $24 \mathrm{~h}$ at room temperature. After that time the solvent was removed under reduced pressure and the residue purified on a silica gel packed column using EtOAc : $n$-hexane (1:1) as an eluent. The products 17a-d were obtained in satisfactory yields.

5-(3-p-Tolyl-1,2,4-oxadiazol-5-yl)pyrimidine-2,4(1H, 3H)-dione (17a). Yield 51\% (0.05 g), mp $196-197{ }^{\circ} \mathrm{C}$ (MeOH). FT IR: 3152, 3051, 1736, 1675, 1620, 1430, 1299, $1016 \mathrm{~cm}^{-1} .{ }^{1} \mathrm{H}$ NMR (300 MHz, DMSO-d 6 ) $\delta(p p m):$ $2.34\left(\mathrm{~s}, 3 \mathrm{H}, \mathrm{Cl}^{\prime}-\mathrm{CH}_{3}\right), 7.26$ (d, 2H, J 8.1, ArH), 7.60 (d, 2H, J $\left.8.1 \mathrm{~Hz}, \mathrm{ArH}\right), 8.74(\mathrm{~s}, 1 \mathrm{H}, \mathrm{H}-6), 12.31(\mathrm{~s}, 1 \mathrm{H}, \mathrm{N} 1-\mathrm{H})$, 
$12.38(\mathrm{~s}, 1 \mathrm{H}, \mathrm{N} 3-\mathrm{H}) .{ }^{13} \mathrm{C} N M R\left(75 \mathrm{MHz}, \mathrm{DMSO}-d_{6}\right) \delta(\mathrm{ppm}): 20.6,89.3,113.5,125.3,127.5,129.1,140.0,142.6$, 147.3, 153.2, 160.0. Anal. Calcd for $\mathrm{C}_{13} \mathrm{H}_{10} \mathrm{~N}_{4} \mathrm{O}_{3}$ (270.24): C, 57.78; $\mathrm{H}, 3.73 ; \mathrm{N}, 20.73 \%$. Found: C, 57.65; $\mathrm{H}, 3.68$; N, $20.59 \%$.

5-(3-(4-Chlorophenyl)-1,2,4-oxadiazol-5-yl)pyrimidine-2,4(1H,3H)-dione (17b). Yield 40\% (0.04 g), $\mathrm{mp} 168-$ $172{ }^{\circ} \mathrm{C}$ (EtOH). FT IR: $3180,3072,2241,1732,1706,1681,1309 \mathrm{~cm}^{-1} .{ }^{1} \mathrm{H}$ NMR $(300 \mathrm{MHz}$, DMSO-d $) \delta(\mathrm{ppm})$ : 7.51 (d, 2H, J 8.4, ArH), 7.76 (d, 2H, J 8.4, ArH), 8.74 (s, 1H, H-6), 12.34 (s, 1H, N1-H), $12.65(\mathrm{~s}, 1 \mathrm{H}, \mathrm{N} 3-\mathrm{H}) .{ }^{13} \mathrm{C}$ NMR $\left(75 \mathrm{MHz}, \mathrm{DMSO}-d_{6}\right) \delta$ (ppm): 89.8, 113.7, 127.4, 128.9, 129.6, 135.1, 142.1, 147.6, 153.4, 160.2. Anal. Calcd for $\mathrm{C}_{12} \mathrm{H}_{7} \mathrm{ClN}_{4} \mathrm{O}_{3}$ (290.66): C, 49.59; $\mathrm{H}, 2.43 ; \mathrm{N}, 19.28 \%$. Found: $\mathrm{C}, 49.37 ; \mathrm{H}, 2.09 ; \mathrm{N}, 19.14 \%$.

5-(3-(4-Bromophenyl)-1,2,4-oxadiazol-5-yl)pyrimidine-2,4(1H, 3H)-dione (17c). Yield 33\% (0.04 g), mp 169$172{ }^{\circ} \mathrm{C}(\mathrm{MeOH})$. FT IR: 3293, 3071, 1768, 1682, 1432, 1297, $1025 \mathrm{~cm}^{-1} .{ }^{1} \mathrm{H}$ NMR $\left(300 \mathrm{MHz}\right.$, DMSO- $\left.d_{6}\right) \delta(\mathrm{ppm}):$ $7.65(\mathrm{~d}, 2 \mathrm{H}, \mathrm{J} 9.0 \mathrm{ArH}), 7.69$ (d, 2H, J 9.0, ArH), 8.74 (s, 1H, H-6), 12.34 (s, 1H, N1-H), $12.66(\mathrm{~s}, 1 \mathrm{H}, \mathrm{N} 3-\mathrm{H}) .{ }^{13} \mathrm{C}$ NMR (75 MHz, DMSO- $\left.d_{6}\right) \delta$ (ppm): 89.8, 113.6, 123.8, 127.6, 129.9, 131.8, 142.2, 147.5, 153.3, 160.2. Anal. Calcd for $\mathrm{C}_{12} \mathrm{H}_{7} \mathrm{BrN}_{4} \mathrm{O}_{3}$ (335.11): C, 43.01; $\mathrm{H}, 2.11 ; \mathrm{N}, 16.72 \%$. Found: $\mathrm{C}, 42.88 ; \mathrm{H}, 1.89 ; \mathrm{N}, 16.68 \%$.

5-(3-(4-Methoxyphenyl)-1, 2, 4-oxadiazol-5-yl)pyrimidine-2, 4(1H, 3H)-dione (17d). Yield 51\% (0.05 g), mp 170$174{ }^{\circ} \mathrm{C}$ (MeOH). FT IR: 3063, 1710, 1657, 1636, 1451, $1318 \mathrm{~cm}^{-1} .{ }^{1} \mathrm{H}$ NMR (300 MHz, DMSO-d $d_{6} \delta$ (ppm): 3.03 (s, $3 \mathrm{H}, \mathrm{OCH}_{3}$ ), 6.99 (d, 2H, J 9.0, ArH), 7.64 (d, 2H, J 9.0, ArH), 8.72 (s, 1H, H-6), $12.22(\mathrm{~s}, 1 \mathrm{H}, \mathrm{N} 1-\mathrm{H}), 12.29(\mathrm{~s}, 1 \mathrm{H}$, N3-H). ${ }^{13} \mathrm{C}$ NMR (75 MHz, DMSO- $d_{6} \delta$ (ppm): 55.1, 89.2, 113.4, 114.0, 122.6, 127.0, 147.2, 153.2, 160.0, 160.7. Anal. Calcd for $\mathrm{C}_{13} \mathrm{H}_{10} \mathrm{~N}_{4} \mathrm{O}_{4}$ (286.24): C, 54.55; H, 3.52; N, 19.57\%. Found: C, 54.35; H, 3.27; N, 19.35\%.

\section{References}

1. Lee, J. M.; Park, S. M.; Kim, B. H. Bioorg. Med. Chem. Lett. 2009, 19, 1126.

http://dx.doi.org/10.1016/j.bmcl.2008.12.103

2. Beale Jr., J. M.; Block, J. H. In Wilson and Gisvold's Textbook of Organic Medicinal and Pharmaceutical Chemistry; Lippincott Williams \& Wilkins, Philadelphia, 2004, p. 391.

3. Semenov, V. E.; Voloshina, A. D.; Toroptzova, E. M.; Kulik, N. V.; Zobov, V. V.; Giniyatullin, R. K.;

Mikhailov, A. S.; Nikolaev,A. E.; Akamsin,V. D.; Reznik, V. S. Eur. J. Med. Chem. 2006, 41, 1093.

http://dx.doi.org/10.1016/j.ejmech.2006.03.030

4. Bohman, C.; Balzarini, J.; Wigerinck, P.; Van Aerschot, A.; Herdewijn, P.; De Clercq, E. J. Biol. Chem. 1994, 269, 8036.

5. Park, S. M.; Yang, H.; Park, S. K.; Kim, H. M.; Kim, B. H. Bioorg. Med. Chem. Lett. 2010, 20, 5831. http://dx.doi.org/10.1016/j.bmcl.2010.07.126

6. Meneni, S.; Ott, I.; Sergeant, C. D.; Sniady, A.; Gust, R.; Dembinski, R. Bioorg. Med. Chem. 2007, 15, 3082. http://dx.doi.org/10.1016/j.bmc.2007.01.048

7. Lee, Y. S.; Park, S. M.; Kim, H. M.; Park, S. K.; Lee, K.; Lee, C. W.; Kim, B. H. Bioorg. Med. Chem. Lett. 2009, $19,4688$.

http://dx.doi.org/10.1016/j.bmcl.2009.06.072

8. Gazivoda, T.; Raić-Malić, S.; Marjanović, M.; Kralj M.; Pavelić K. Bioorg. Med. Chem., 2007, 15, 749. http://dx.doi.org/10.1016/j.bmc.2006.10.046

9. Sari, O.; Roy, V.; Balzarini, J.; Snoeck, R.; Andrei, G.; Agrofoglio, L. A. Eur. J. Med. Chem. 2012, 53, 220. http://dx.doi.org/10.1016/j.ejmech.2012.04.001

10. De Clercq, E.; Nucleos. Nucleot. 1994,13, 1271. http://dx.doi.org/10.1080/15257779408012151 
11. Olivier, A.; Creuven, I.; Evrard, C.; Evrard, G.; Dory, M.; van Aerschot, A.; Wigerinck, P.; Herdewijn, P.; Durant, F. Antiviral Res. 1994, 24, 289.

http://dx.doi.org/10.1016/0166-3542(94)90076-0

12. Creuven, I.; Evrard, C.; Olivier, A.; Evrard, G.; Van Aerschot, A.; Wigerinck, P.; Herdewijn, P.; Durant, F. Antiviral Res. 1996, 30, 63.

http://dx.doi.org/10.1016/0166-3542(95)00838-1

13. Wigerinck, P.; Pannecouque, C.; Snoeck, R.; Claes, P.; De Clercq, E.; Herdewijn, P. J. Med. Chem. 1991, 34, 2383.

http://dx.doi.org/10.1021/jm00112a011

14. Wigerinck, P.; Kerremans, L.; Claes, P.; Snoeck, R.; Maudgal, P.; De Clercq, E.; Herdewijn, P. J. Med. Chem. 1993, 36, 538.

http://dx.doi.org/10.1021/jm00057a003

15. Balzarini, J.; McGuigan, Ch. J. Antimicrob. Chem. 2002, 50, 5.

http://dx.doi.org/10.1093/jac/dkf037

16. Herdewijn, P. A. M. M. Antiviral Chem. Chemother. 1994, 5,131.

http://dx.doi.org/10.1177/095632029400500301

17. McGuigan, C.; Barucki, H.; Carangio, A.; Blewett, S.; Andrei, G.; Erichsen, J. T.; Snoeck, R.; De Clercq, E.; Balzarini, J. J. Med. Chem. 2000, 43, 4993.

http://dx.doi.org/10.1021/jm000210m

18. McGuigan, C.; Pathirana, R. N.; Jones, G.; Andrei, G.; Snoeck, R.; De Clercq, E.; Balzarini, J. Antiviral Chem. Chemother. 2000, 11, 343.

http://dx.doi.org/10.1177/095632020001100505

19. Carangio, A.; Srinivasan, S.; McGuigan, C.; Andrei, G.; Snoeck, R.; De Clercq, E.; Balzarini, J. Antiviral Chem. Chemother. 2002, 13, 263.

http://dx.doi.org/10.1177/095632020201300501

20. Onishi, T.; Mukai, C.; Nakagawa, R.; Sekiyama, T.; Aoki, M.; Suzuki, K.; Nakazawa, H.; Ono, N.; Ohmura, Y.; Iwayama, S.; Okunishi, M.; Tsuji, T. J. Med. Chem. 2000, 43, 278.

http://dx.doi.org/10.1021/jm9904194

21. Carangio, A.; McGuigan, C.; Andrei, G.; Snoeck, R.; De Clercq, E.; Balzarini, J. Antivir. Chem. Chemother. 2001, 12, 187.

http://dx.doi.org/10.1177/095632020101200306

22. Ivanov, A. V.; Simonian, A. R.; Belanov, E. F.; Aleksandrova, L. A. Russ. J. Bioorg. Chem. 2005, $31,556$. http://dx.doi.org/10.1007/s11171-005-0076-7

23. Migliore, M. Antivir. Chem. Chemother. 2010, 20, 107. http://dx.doi.org/10.3851/IMP1472

24. Robins, M. J.; Miranda, K.; Rajwanshi, V. K.; Peterson, M. A.; Andrei, G.; Snoeck, R.; De Clercq, E.; Balzarini, J. J. Med. Chem. 2006, 49, 391.

http://dx.doi.org/10.1021/jm050867d

25. Balzarini, J.; McGuigan, C. Biochim. Biophys. Acta. 2002, 1587, 287. http://dx.doi.org/10.1016/S0925-4439(02)00091-1

26. McGuigan, C.; Yarnold, C. J.; Jones. G.; Velázquez, S.; Barucki, H.; Brancale, A.; Andrei, G.; Snoeck, R.; De Clercq, E.; Balzarini, J. J. Med. Chem. 1999, 42, 4479.

http://dx.doi.org/10.1021/jm990346o 
27. Andronova, V. L.; Skorobogaty̆̆, M. V.; Manasova, E. V.; Berlin, lu. A.; Korshun, V. A.; Galegov, G. A. Russ. J. Bioorg. Chem. 2003, 29, 262.

\section{http://dx.doi.org/10.1023/A:1023936516589}

28. Robins, M. J.; Nowak, I.; Rajwanshi, V. K.; Miranda, K.; Cannon, J. F.; Peterson, M. A.; Andrei, G.; Snoeck, R.; De Clercq, E.; Balzarini. J. J. Med. Chem. 2007, 50, 3897.

http://dx.doi.org/10.1021/jm070210n

29. Fan, X.; Zhang, X.; Zhou, L.; Keith, K.A.; Kern, E. R.; Torrence, P. F. J. Med. Chem. 2006, 49, 3377. http://dx.doi.org/10.1021/jm0601710

30. Abdel-Rahman, A. A.; Wada, T. Naturforsch. C, 2009, 64, 163.

31. Park, J. S.; Chang, C. T.; Schmidt, C. L.; Golander, Y.; De Clercq, E.; Descamps, J.; Mertes, M. P. J. Med. Chem. 1980, 23, 661.

http://dx.doi.org/10.1021/jm00180a016

32. Janeba, Z.; Balzarini, J.; Andrei, G.; Snoeck, R.; De Clercq, E.; Robins, M. J. J. Med. Chem. 2005, 48, 4690. http://dx.doi.org/10.1021/jm050291s

33. Fan, X.; Zhang, X.; Bories, C.; Loiseau, P. M.; Torrence, P. F. Bioorg. Chem. 2007, 35, 121. http://dx.doi.org/10.1016/j.bioorg.2006.08.004

34. Van Poecke, S.; Sinnaeve, D.; Martins, J. C.; Balzarini, J.; Van Calenbergh, S. Nucleos. Nucleot. Nucl. 2012, 31, 256.

35. Montagu, A.; Roy, V.; Balzarini, J.; Snoeck, R.; Andrei, G.; Agrofoglio, L, A. Eur. J. Med. Chem. 2011, 46, 778.

http://dx.doi.org/10.1016/j.ejmech.2010.12.017

36. Wigerinck, P.; Snoeck, R.; Claes, P.; De Clercq, E.; Herdewijn, P. J. Med. Chem., 1991, 34, 1767. http://dx.doi.org/10.1021/jm00110a003

37. Kumar, R.; Rai, D.; Sharma, S.K.; Saffran H. A.; Blush, R.; Tyrrell, D. L. J. Med. Chem. 2001, 44, 3531. http://dx.doi.org/10.1021/jm010226s

38. Torrence, P. F.; Fan, X.; Zhang, X.; Loiseau, P. M. Bioorg. Med. Chem. Lett. 2006, 16, 5047. http://dx.doi.org/10.1016/j.bmcl.2006.07.042

39. Fan, X.; Zhang, X.; Bories, C.; Loiseau, P. M.; Torrence, P. F. Bioorg Chem. 2007, 35, 121. http://dx.doi.org/10.1016/j.bioorg.2006.08.004

40. Miller, L. F.; Bambury, R. E. J. Med. Chem. 1970, 13, 1022.

http://dx.doi.org/10.1021/jm00299a072

41. Ressner, E. C.; Fraher, P.; Edelman, M. S.; Mertes, M. P. J. Med. Chem. 1976, 19, 194. http://dx.doi.org/10.1021/jm00223a042

42. Ross, L. O.; Acton, E. M.; Skinner, W. A.; Goodman, L.; Baker, B. R. J. Org. Chem. 1961, 26, 3395. http://dx.doi.org/10.1021/jo01067a092

43. Pesnot, T.; Tedaldi, L. M.; Jambrina, P. G.; Rostac, E.; Wagner, G. K. Org. Biomol. Chem. 2013, $11,6357$. http://dx.doi.org/10.1039/c3ob40485d

44. Fresneau, N.; Hiebel, M. A.; Agrofoglio, L. A.; Berteina-Raboin, S. Molecules 2012, 17, 14409. http://dx.doi.org/10.3390/molecules171214409

45. Herve, G.; Sartori, G.; Enderlin, G.; Mackenzie, G.; Len, Ch. RSC Adv. 2014, 4, 18558.

46. Jakubiec, D.; Walczak, K. Z. in Targets in Heterocyclic systems. Chemistry and Properties. Reviews and Accounts on Heterocyclic Chemistry; Attanasi, A. O.; Spinelli, D. Eds.; Rome: Societa Chimica Italiana, 2012, Vol. 16, p. 128.

47. Mendonca, F. J. B.; dos Anjos, J. V.; Sinou, D.; de Melo, S. J.; Srivastava, R. M. Synthesis, 2007, $12,1890$. 
48. Kocalka, P.; Andersen, N. K.; Jensen, F.; Nielsen, P. Chem. Bio. Chem. 2007, 8, 2106. http://dx.doi.org/10.1002/cbic.200700410

49. Dodd, D. W.; Swanick, K. N.; Price, J. T.; Brazeau, A. L.; Ferguson, M. J.; Jones, N. D.; Hudson, R. H. E. Org. Biomol. Chem., 2010, 8, 663.

http://dx.doi.org/10.1039/B919921G

50. Shaikh, K. I.; Madsen, C. S.; Nielsen, L. J. Joergensen, A. S.; Nielsen, H.; Petersen, M.; Nielsen, P. Chem. Eur. J. 2010, 16, 12904. http://dx.doi.org/10.1002/chem.201001253

51. Stephenson, A. W. I.; Partridge, A. C.; Filichev, V. V. Chem. Eur. J. 2011, 17, 6227. http://dx.doi.org/10.1002/chem.201003200

52. Coutouli-Argyropoulou, E.; Lianis, P.; Mitakou,M.; Giannoulisa, A.; Nowak, J. Tetrahedron 2006, 62, 1494. http://dx.doi.org/10.1016/i.tet.2005.11.019

53. Jakubiec, D.; Walczak, K. Z. Tetrahedron Lett. 2011, 52, 6890.

http://dx.doi.org/10.1016/j.tetlet.2011.10.033

54. Coutouli-Argyropoulou, E.; Trakossas, S. Tetrahedron 2011, 67, 1915.

http://dx.doi.org/10.1016/j.tet.2011.01.020

55. Hirota, K.; Kitade, Y.; Shimada, K.; Maki, Y. J. Org. Chem. 1985, 50, 1512.

http://dx.doi.org/10.1021/jo00209a030

56. Chiacchio, U.; Corsaro, A.; Mates, J.; Merino, P.; Piperno, A.; Rescifina, A.; Romeo, G.; Romeo, R.; Tejero, T. Tetrahedron 2003, 59, 4733. http://dx.doi.org/10.1016/S0040-4020(03)00689-6

57. Boncel, S.; Osyda, D.; Walczak, K. Z. Beilstein J. Org. Chem. 2007, 3, 40. http://dx.doi.org/10.1186/1860-5397-3-40

58. Giurg, M.; Młochowski, J. Pol. J. Chem. 1997, 71, 1093. 